Mannheimer Manuskripte zu Risikotheorie,

Portfolio Management und Versicherungswirtschaft

\author{
Nr. 150 \\ Z um fairen Wert der Aktienanlagen eines \\ L ebensversicherungsunternehmens \\ aus ökonomisch-statistischer Perspektive
}

von

Peter Albrecht

Mannheim 09/2003 


\section{Zum fairen Wert der Aktienanlagen eines \\ Lebensversicherungsunternehmens aus ökonomisch-statistischer Perspektive}

Prof. Dr. Peter Albrecht

Institut für Versicherungswissenschaft

Universität Mannheim

(Version: 01.09.03) 


\section{Inhaltsverzeichnis}

1. Einführung und Problemstellung

2. Theoretische Grundlagen zur Ermittlung eines fairen Wertes

2.1 Anforderungen an einen "fairen" Wert

2.2 Ausgangspunkt: Going Concern-Prinzip

2.3 Martingal-Hypothese

2.4 Random Walk-Hypothese

2.5 Mean Reversion-Hypothese

3. Die Entwicklung des DAX-KGV

4. Eine DAX-Projektion

5. Langfristcharakter des Versicherungsgeschäfts und Bewertung

6. Zusammenfassung und Schlussfolgerungen

Anhang A: Modellgrundlagen

A.1: Beste Prädiktion

A.2: Martingal-Hypothese

A.3: Random Walk-Hypothese

A.4: Mean Reversion-Hypothese

Anhang B: Statistische Analyse der DAX-KGV-Zeitreihe

B.1 Weitergehende explorative Analyse

B.2 Spezifikation und Analyse der Basis-Regressionsgleichung

Anhang C: Grundlagen einer DAX-Projektion

Literaturverzeichnis 


\section{Einführung und Problemstellung}

Der in den Jahren 2000 - 2002 eingetretene Kursverfall des DAX hat den Wert eines DAXPortfolios in diesem Zeitraum mehr als halbiert ${ }^{1}$. Vor diesem Hintergrund stellt sich die Frage, welche Werthaltigkeit dem Aktienengagement eines Lebensversicherungsunternehmens beizumessen und welcher Wertmaßstab zur Einschätzung eines "fairen Wertes" zu verwenden ist. Dieser Fragestellung soll in diesem Beitrag in grundsätzlicher Weise nachgegangen werden, d.h. zunächst ${ }^{2}$ ohne Bezug auf die konkret gültigen Rechnungslegungsvorschriften.

Die gestellte Problematik führt dabei auf eine Reihe von weiteren zentralen Problemkreisen. Zunächst ist die Frage zu klären, welche Anforderungen an die Spezifikation eines "fairen Wertes" aus modelltheoretischer Perspektive zu stellen sind. Dann stellt sich die Frage der anzuwendenden Bewertungsperspektive, konkret geht es um die Perspektiven "Unternehmenszerschlagung" bzw. "dauerhafte Unternehmensfortführung" (Going Concern-Hypothese). Des Weiteren ist dem Problemkreis nachzugehen, welche Modelle aus ökonomischstatistischer Perspektive zur Abbildung der Zufallsdynamik von Aktienkursen angemessen sind. Insbesondere führt dies zur Fragestellung, ob eher die Random Walk-Hypothese oder eher die Mean Reversion-Hypothese den Kursverlauf auf Aktienmärkten angemessen repräsentiert. Die Beantwortung dieser Frage geschieht dabei unter Rückgriff auf neuere ${ }^{3}$ statistische Evidenz hinsichtlich des Vorliegens von Mean Reversion-Effekten für die Wertentwicklung des Kurs/Gewinn-Verhältnisses des deutschen Aktienindex (DAX). Da sich solche Mean Reversion-Effekte nicht notwendigerweise über kurze oder auch mittelfristige Zeiträume einstellen müssen, ist schließlich der Frage nachzugehen, inwieweit Lebensversicherungsunternehmen auch längere Phasen von Bewertungsuntertreibungen durchstehen können, ohne dabei eine vorzeitige Liquidation des Aktienportfolios vornehmen zu müssen.

Sämtliche vorstehend dargelegten Problemkreise werden im Folgenden eingehend erörtert.

1 Die realisierte (nicht-annualisierte) Gesamtrendite des DAX über diese drei Jahre entspricht einem Wertverlust von 58\%, vgl. im Einzelnen etwa Albrecht (2003).

2 Die Folgerungen im Rahmen eines De lege lata-Ansatzes sollen in einem separaten Beitrag gezogen werden.

$3 \quad$ Vgl. Albrecht/Kantar(2003). 


\section{Theoretische Grundlagen zur Ermittlung eines fairen Wertes}

\subsection{Anforderungen an einen "fairen" Wert}

Der Terminus "fairer" Wert wird in der wissenschaftlichen Literatur höchst unterschiedlich verstanden und konkretisiert ${ }^{4}$. Es ist nicht das Ziel der vorliegenden Ausarbeitung, hierzu eine kritische Literaturdiskussion zu führen, die auf eine einheitliche Begriffsbildung abzielt. Es genügt, die Anforderungen an einen "fairen Wert" im vorliegenden Kontext der Bewertung eines Aktienportfolios zu spezifizieren. Zunächst ist dies die

\section{(A) Anforderung der Modellgestütztheit.}

Im vorliegenden ökonomischen Kontext der Bewertung auf Kapitalmärkten dienen als relevante Modelle vor allem ökonomische Gleichgewichtsmodelle unter Risiko.

Als zweite Bedingung stellen wir die

(B) Anforderung der statistischen Repräsentanz.

Dies betrifft sowohl die Frage der statistischen Identifizierbarkeit des ökonomischen Modells als auch die Eigenschaft, dass das Modell die Daten angemessen repräsentiert und damit auf der Grundlage des verwendeten Modells auch Aussagen über die Realität getroffen werden können.

Schließlich soll auf im Rahmen eines ökonomisch begründeten und statistisch angemessenen Modells der dem Aktienportfolio beizulegende Wert die vorhandenen Informationen "bestmöglich" widerspiegeln. Damit kommen wir zur

(C) Anforderung der bestmöglichen Werteinschätzung.

4 Um nur einige Beispiele, ohne jeglichen Anspruch auf Vollständigkeit, zu nennen: Im Rechnungswesen existieren die Konzeptionen des "Fair Value Accounting" und des "Fair Value", die noch Gegenstand der weiteren Ausführungen sein werden. Von "actuarially fair" spricht man bei Anwendung des Äquivalenzprinzips. Von einem "fairen Spiel" ("fair game") spricht man bei Vorliegen der Martingaleigenschaft, vgl. hierzu Anhang A.2. In der ökonomischen Literatur spricht man von einem fairen Wert, wenn dieser das Ergebnis eines Gleichgewichtsmodells ist (z.B. des Capital Asset Pricing-Modells oder eines optionspreistheoretischen Modells - um nur zwei Beispiele zu nennen). 
Modelltheoretisch führt ${ }^{5}$ diese Anforderung auf die Theorie der besten Prädiktion bzw. des besten Prädiktors. Diese ist - unter der Standardannahme, dass als (zu minimierendes) Gütekriterium der mittlere quadratische Fehler (Mean Square Error, MSE) verwendet wird - in Anhang A.1 kurz dargestellt.

Die im Weiteren diskutierten Modelle, nämlich Martingal, Random Walk sowie AR(1)Prozess, sind die finanzmathematischen Standardmodelle, mit denen die Zufallsdynamik der Entwicklung von Aktienportfolios, Aktienindices sowie Einzelaktien beschrieben wird. Alle diese Modelle können sowohl ökonomisch fundiert ${ }^{6}$ als auch statistisch identifiziert ${ }^{7}$ werden. Ebenso sind im Rahmen dieser Modelle jeweils bestmögliche Werteinschätzungen im Sinne der besten Prädiktion möglich. Alle drei in den folgenden Abschnitten eingehender behandelten Modelle sind daher nicht nur finanzmathematische Standardmodelle, sondern erfüllen auch sämtliche der postulierten Anforderungen.

Zuvor ist aber zunächst einmal die Bewertungsperspektive abzuklären.

\subsection{Ausgangspunkt: Going Concern-Prinzip}

Grundsätzlicher Ausgangspunkt der im Weiteren durchgeführten Analysen zur Ermittlung eines fairen Wertes des Aktienportfolios eines Unternehmens ist die Prämisse der Fortführung der Unternehmenstätigkeit (Going Concern-Prinzip). Unter der Alternativprämisse, der Unternehmensliquidation (Unternehmenszerschlagung) müssen die Aktiva des Unternehmens zum jeweiligen Marktwert veräußert werden, insofern ist eine Marktbewertung unter der Perspektive einer Unternehmenszerschlagung nur konsequent.

Die Einnahme einer solchen Perspektive ist jedoch primär ein relevanter Gesichtspunkt im Rahmen einer Liquiditätsanalyse ${ }^{8}$. Im Hinblick auf die Erstellung eines Jahresabschlusses ist

5 Dabei wird wesentlich von einem prospektiven Verständnis der Informationsfunktion der Rechnungslegung ausgegangen.

6 Das Martingalmodell wird üblicherweise im Kontext der Markteffizienzthese gesehen, vgl. für einen Überblick etwa LeRoy (1989). (Multiplikativer) Random Walk bzw. AR(1)-Prozess werden in der Kapitalmarkttheorie zumeist in ihrer zeitstetigen Spezifizierung als Geometrische Brownsche Bewegung, vgl. hierzu etwa Albrecht/Maurer (2002, S. 148 ff.), bzw. als Ornstein/Uhlenbeck-Prozess, vgl. ebenda, S. 152 f., betrachtet. Im Rahmen der vorliegenden Arbeit gehen wir dabei jeweils von der allgemeinen zeitdiskreten Variante aus.

7 Für das Martingal vgl. hierzu etwa LeRoy (1989, S. 1895 ff.). Für Random Walk bzw. AR(1)-Prozess vgl. die Ausführungen im Rahmen der weiteren Arbeit sowie Albrecht/Kantar (2003).

8 Vgl. hierzu etwa Coenenberg (2003, S. 962) oder Küting/Weber (2001, S. 79). 
hingegen der Grundsatz der Fortführung der Unternehmenstätigkeit ein im Handelsgesetzbuch $^{9}{ }^{10}$ kodifizierter Grundsatz ordnungsgemäßer Buchführung ${ }^{11}$. Nach dem Going Concern-Prinzip ist demnach bei der Bewertung der Vermögenswerte und Schulden im Jahresabschluss von der Prämisse auszugehen, dass das Unternehmen über den Abschlussstichtag hinaus fortgeführt wird.

Küting/Weber (2003, S. 159) schreiben hierzu:

"Die Bewertung in der Handelsbilanz soll sich demnach ... an der betrieblichen Realität der Unternehmensfortführung orientieren. Solange die Fortführung des Unternehmens tatsächlich beabsichtigt und rechtlich möglich ist, sind die Vermögenswerte infolgedessen gem. ihrer tatsächlich beabsichtigen Verwendung im normalen Leistungsproze $\beta$ des Unternehmens zu bewerten. ... $\S 252$ Abs. 1 Nr. 2 verbietet damit grundsätzlich die auf Vorsichtsüberlegungen basierende oder durch Zweifel "begründete" Unterstellung einer Unternehmenszerschlagung."

Da im Zentrum des vorliegenden Beitrags jedoch nicht die Vornahme einer Liquiditätsanalyse oder die Aufstellung eines Liquiditätsplans steht, sondern die Ermittlung eines fairen Wertes des Aktienportfolios eines Lebensversicherungsunternehmens bei Fortführung der normalen Unternehmenstätigkeit, bildet das Going Concern-Prinzip die Prämisse der weiteren Analysen.

\subsection{Martingal-Hypothese}

Genügt die Wertentwicklung eines Aktienportfolios oder eines Aktienindex, wie etwa des DAX, der Martingal-Hypothese ${ }^{12}$, so stellt der heutige bzw. der zuletzt beobachtete Wert den bestmöglichen Prognosewert ${ }^{13}$ für den Wert zu einem (beliebigen) künftigen Zeitpunkt dar. Der heutige Marktwert bzw. der Marktwert zum Bilanzstichtag würde in diesem Falle dem fairen Wert des Aktienportfolios auch aus ökonomisch-statistischer Sicht entsprechen. Der Marktwert würde in diesem Falle auch jenseits seiner Eigenschaft als Zerschlagungswert, d.h. auch unter Anwendung der Going Concern-Hypothese, eine ökonomisch begründbare Konkretisierung der Konzeption eines fairen Wertes darstellen.

$9 \quad$ Vgl. Par. 252 (1) HGB.

10 Auch im Rahmen der Rechnungslegungsgrundsätze nach IAS zählt das Going Concern-Prinzip zu den Grundannahmen (underlying assumptions), vgl. hierzu Coenenberg (2003, S. 56).

11 Vgl. hierzu etwa Coenenberg (2003, S. 45) oder Küting/Weber (2001, S. 195).

$12 \mathrm{Vgl}$. zu den formalen Aspekten die Ausführungen in Anhang A.2.

13 Vgl. zu den formalen Aspekten die Darstellung in Anhang A.1. 
Die MartingalHypothese wird oftmals in engem Zusammenhang mit der Hypothese der $\mathrm{Ka}$ pitalmarkteffizienz $^{14}$ gesehen. In einem effizienten Kapitalmarkt reflektieren (grob gesagt) die heutigen Preise bereits "alle"15 den Investoren am Markt verfügbaren Informationen (Informationseffizienz). Empirische Tests der Kapitalmarkteffizienz laufen in der Regel ${ }^{16}$ auf Tests der Martingal-Hypothese hinaus. Allerdings ist dieser Übergang, wie LeRoy (1989) als Résumé17 seiner Ausarbeitung zieht, mit einer Reihe von Problemen behaftet. Für die Zwecke der vorliegenden Ausarbeitung sind allerdings die genauen Zusammenhänge zwischen Martingal Hypothese und Effizienzthese weniger von Belang. Es genügt zu klären, ob die Martingal Hypothese theoretisch sowie empirisch Bestand hat.

Aus theoretischer Sicht ist inzwischen geklärt, dass die Martingal-Hypothese nicht aufrecht erhalten werden kann. Der "Beweis" der Martingaleigenschaft der Preise von Finanztiteln durch Samuelson $(1965,1973)$ beruht essentiell ${ }^{18}$ auf der kritischen Annahme der Risikoneut tralität der Investoren. Lässt man diese Annahme fallen, so zeigt sich, dass im Rahmen allgemeiner Gleichgewichtsmodelle Preise resultieren, die die MartingalHypothese verletzen ${ }^{19}$.

Campbell et al. (1997, S. 31) formulieren hierzu:

"Therefore, despite the intuitive appeal that the fair-game interpretation might have, it has been shown that the martingale property is neither a necessary nor a sufficient condition for rationally determined asset prices."

Auch aus empirischer Sicht ${ }^{20}$ ist die Martingal-Hypothese nicht haltbar. Aktienkurse weisen typischerweise eine positive Drift auf (dies liegt begründet in dem Wachstum der zugrundeliegenden Volkswirtschaft), damit ist die Martingaleigenschaft bereits verletzt ${ }^{21}$. Aus empirischer Sicht liegt somit ein Submartingal ${ }^{22}$ vor, entsprechend wird im Zusammenhang mit der

14 Zur Kapitalmarkteffizienz vgl. etwa Campbell et al. (1997, S. 20 ff.), Lo (1996) oder Steiner/Bruns (2002, S. 41 ff.).

$15 \mathrm{Zu}$ den verschiedenen Varianten der Effizienzthese in Termen unterschiedlicher Informationsmengen vgl. etwa Campbell et al. (1997, S. 22).

16 Vgl. zu dieser Aussage LeRoy (1989, S. 1613).

17 Vgl. ebenda.

18 Vgl. ebenda, S. 1603.

19 Die fundamentalen Beiträge hierzu sind LeRoy (1973) und Lucas (1978), man vgl. auch die Übersicht in LeRoy (1989, S. 1603 ff.).

20 Vgl. hierzu etwa die Übersicht in LeRoy (1989, S. 1595 ff.) sowie die in Abschnitt 2.4 zitierte Literatur zu Mean Reversion-Tendenzen auf Aktienmärkten.

21 Vgl. hierzu die Eigenschaften eines Martingals in Anhang A.2.

22 Vgl. hierzu Anhang A.2. 
Hypothese der Kapitalmarkteffizienz auch die Submartingal-Variante ins Spiel gebracht ${ }^{23}$. Bei Vorliegen eines Submartingals ist der aktuelle Wert aber nicht mehr der beste Prädiktor für die künftige Wertentwicklung.

Fassen wir zusammen: Bei Gültigkeit der Martingal-Hypothese für die Entwicklung von Aktienkursen wäre der Marktwert der beste Prognosewert für den Wert des Aktienportfolios zu einem (beliebigen) künftigen Zeitpunkt. Damit wäre der Marktwert im Rahmen eines Going Concern-Ansatzes eine ökonomisch begründbare Spezifikation eines fairen Wertes. Wie ausgeführt, ist aber die Martingal-Hypothese weder theoretisch noch empirisch haltbar. Damit verliert der Marktwert aber seine Begründungsbasis im Rahmen von ökonomischen (Gleichgewichts-)Modellen ${ }^{24}$. Dies zeigt zugleich, dass das Verständnis des Marktwertes als Fair Value gemäß der nationalen und internationalen Rechnungslegungsnormen ${ }^{25}$ nicht notwendigerweise ein Fair Value im Sinne eines ökonomischen Gleichgewichtsansatzes ist, sondern sich das Attribut fair primär auf die Objektivierbarkeit des beizulegenden Wertes (Orientierung an Markttransaktionen und nicht an der individuellen Einschätzung) bezieht. Dies ist aber ein fundamentaler Unterschied und relativiert damit die Begründungsbasis für die (internationale) Vorherrschaft des Fair Value-Paradigmas erheblich. Gerade der dramatische Kursverfall der Aktienmärkte in den Jahren 2000 - 2002 bestätigt eindrucksvoll, dass die Marktwerte z.B. des Jahres 1999 keinesfalls faire Widerspiegelungen des wahren Wertes der zugrundeliegenden Aktien gewesen sind, sondern im Gegenteil eher erhebliche Informationsverzerrungen beinhaltet haben. Insofern erscheint die z.B. von Coenenberg (2003, S. 107) getroffene Übersetzung des Fair Value als "beizulegender Zeitwert" vollständig angemessen und entmystifiziert den Fair Value in begrüßenswerter Weise.

Vgl. hierzu ursprünglich Fama (1976) sowie etwa Perridon/Steiner (2002, S. 223) oder Steiner/Bruns (2002, S. 43).

24 Zugleich zeigt dies, dass die in Coenenberg (2003, S. 107) zu findende Charakterisierung, dass das Ziel einer Fair Value-Bewertung "die Bündelung der am Markt bezüglich des Bewertungsobjekts vorhandenen Informationen in der Rechnungslegung" sei, sich nur auf die aus diesen Informationen resultierenden Marktpreise bezieht und nicht auf eine Bündelung dieser Informationen im Sinne einer besten Prädiktion.

25 Zur Fair Value-Konzeption gemäß HGB, IAS und US-GAAP vgl. eingehend Coenenberg (2003, S. 107 ff.). Unter dem Fair Value eines Vermögenswertes oder einer Schuld wird dabei grundsätzlich derjenige Betrag verstanden, zu dem zwei voneinander unabhängige Parteien mit Sachverstand und Abschlusswillen bereit wären, das Asset zu tauschen bzw. die Liability zu begleichen. Vorausgesetzt wird dabei, dass die Transaktion unter marktüblichen Bedingungen (arm's length transaction) und ohne Abschlusszwang erfolgt. Sofern die zu bewertenden Positionen auf Märkten gehandelt werden, wird dabei häufig der Marktwert als Anhaltspunkt für die Bestimmung des Fair Value herangezogen. 


\section{$2.4 \quad$ Random Walk-Hypothese}

Das wahrscheinlichkeitstheoretisch-statistische Basismodell zur Modellierung von Aktienkursentwicklungen ist der Random Walk ${ }^{26}$ (mit Drift ${ }^{27}$ ). Die Gültigkeit der Random WalkHypothese hat eine Reihe von zentralen Konsequenzen für die Prognostizierbarkeit (Predictability) der künftigen Aktienkursentwicklung. Diesen Konsequenzen soll im Folgenden näher nachgegangen werden.

Zunächst einmal sind im Falle des Vorliegens eines Random Walk wie beim Martingal die Wertveränderungen aufeinanderfolgender Perioden nicht korreliert, d.h. es besteht diesbezüglich kein (linearer) statistischer Zusammenhang. Entscheidend - und auch im Unterschied zum Martingal - für die Prognostizierbarkeit ist aber eine zweite Eigenschaft ${ }^{28}$ des Random Walk, die mögliche Aufschaukelung von Fehlern (Schocks). Dies führt dazu, dass die Abweichungen des Random Walk von seinem Basistrend in systematischer Hinsicht beliebig groß werden können. Shiller/Perron (1985, S. 381) charakterisieren diese Random WalkEigenschaft folgendermaßen:

"In the finance context, the random walk null hypothesis means that price $p_{t}$ can never be described as "too high" (i.e., that it can be expected to fall in the future) or "too low" (i.e., that it can be expected to rise in the future)."

Insofern ist die erwartete Entwicklung keine zuverlässige Prognose für die sich künftig realisierende Entwicklung. Das Random Walk-Modell beinhaltet daher im Kern eine NichtPrognostizierbarkeit der künftigen Wertveränderungen.

Damit lässt sich zugleich der Ansatz des Marktwertes zur Bewertung eines Aktienportfolios nicht auf der Basis der Random Walk-Hypothese begründen. Über die Eigenschaft als fairer im Sinne eines objektivierbaren Wertes hinaus besteht hier keine weitergehende Substantiierung der Vorzugswürdigkeit des Marktwertes auf der Basis einer ökonomischen oder statistischen Modelleigenschaft.

Allerdings existiert bei Vorliegen eines Random Walk auch kein anderer Wert, dem die Eigenschaft eines fairen Wertes im Sinne einer ökonomischen Modellierung zugeschrieben

26

Grundsätzlich zum Modell des Random Walk vgl. Albrecht/Maurer (2002, S. 140 ff.) sowie die Ausführungen in Anhang A.3.

27 der Martingaleigenschaft.

28 Vgl. zu den formalen Aspekten Anhang A.3. 
werden kann. Zumindest aus ökonomisch-statistischer Modellperspektive ist somit das Vorliegen eines Random Walks etwas misslich. Aus pragmatischen Gründen würde man sich dann doch wieder auf den Marktwert zurückziehen müssen, denn dieser erfüllt zumindest das Gütekriterium der Objektivierbarkeit und es existiert kein alternativer Wert, dem überlegene Güteeigenschaften zugeschrieben werden können.

\subsection{Mean Reversion-Hypothese}

Ein alternatives wahrscheinlichkeitstheoretisch-statistisches Modell ist der autoregressive Prozess erster Ordnung, kurz AR(1)-Prozess ${ }^{29}$, der im Weiteren als Basismodell für die Erfassung eines Mean Reversion-Effektes dient.

AR(1)-Prozesse weisen ein langfristiges Mittel auf, der Wertverlauf des Prozesses wird bei temporären Abweichungen mit einer gewissen Elastizität auf dieses Langfristmittel hingezogen. Im Unterschied zum Random Walk kann es damit nicht in systematischer Hinsicht zu beliebig großen Abweichungen vom zugrundeliegenden Trend kommen. In Termen der besten Prädiktion ${ }^{30}$ (im Sinne des mittleren quadratischen Fehlers) impliziert dies, dass der Einfluss der momentanen Abweichung vom Langfristmittel (mit geometrischer Rate) abnimmt und sich der bestmögliche Prognosewert mit steigender Länge des Projektionshorizonts immer mehr dem Langfristmittel annähert.

Die (empirische) Gültigkeit der Mean Reversion-Hypothese - repräsentiert durch einen AR(1)-Prozess - hat offenbar dramatische Auswirkungen in bezug auf das Paradigma des "Fair Value-Accounting" bzw. auf die im (internationalen) Rechnungswesen vorherrschende Marktwertsicht. Bei Validität der Mean Reversion-Hypothese ist nicht der Marktwert der beste Prädiktor für den "fairen Wert", sondern das Langfristmittel des AR(1)-Prozesses. Der Marktwert als dominanter Bewertungsmaßstab wäre damit nachhaltig erschüttert ${ }^{31}$.

$\mathrm{Da}$ - wie in Abschnitt 2.3 ausgeführt - die Martingal-Hypothese weder theoretisch noch empirisch haltbar ist, spitzt sich die Frage nach der Spezifikation eines fairen Wertes, zu dem ein

29 Zu den formalen Aspekten eines AR(1)-Prozesses vgl. etwa Hamilton (1994, S. 53 ff.) sowie die Ausführungen in Anhang A.4

30 Vgl. zu den formalen Aspekten Anhang A.4.

31 So auch McCarthy (2003, S. 93) in seiner Besprechung zu Campbell/Viceira (2002): „The evidence of this book seems to support the fact that the equity markets do have some element of mean reversion, posing a challenge for fair-value accounting gurus." 
Aktienportfolio bewertet werden kann, somit zu auf die Frage "Random Walk oder Mean Reversion?". Im ersten Falle wäre - wenn auch mehr aus pragmatischer Sicht ${ }^{32}-$ der Marktwert anzusetzen, im letzteren Falle wäre das Langfristmittel des AR(1)-Prozesses die ökonomischstatistisch begründete Spezifikation eines fairen Wertes.

Zur Frage "Random Walk oder Mean Reversion?" existieren inzwischen eine Reihe von wissenschaftlichen Untersuchungen. Fama/French (1988), Lo/MacKinlay (1988) sowie Poterba/Summers (1988) waren die ersten Beiträge, in denen Hinweise dokumentiert wurden, dass zwar über kurze und mittlere Frist die Random Walk-Hypothese für die Modellierung von Kursentwicklungen auf Aktienmärkten angemessen ist, über lange Zeithorizonte jedoch Mean Reversion-Effekte in Aktienkurszeitreihen existieren. Die Ergebnisse dieser frühen Arbeiten wurden in jüngerer Zeit erhärtet durch die Beiträge von Balvers/Wu/Gilliland (2000), Campbell/Viceira (1999, 2002) sowie für den deutschen Aktienmarkt aktuell von Eberts (2003).

Im Unterschied zu den vorstehenden Arbeiten, bei der die Analysen auf der reinen Kurs- bzw. Renditeebene durchgeführt wurden, wird in dem Beitrag von Albrecht/Kantar (2003) eine entsprechende Analyse auf Fundamentalebene in Termen der Entwicklung des Kurs/GewinnVerhältnisses $(\mathrm{KGV})$ durchgeführt ${ }^{33}$

Eine Analyse auf der KGV-Ebene ist aus unserer Sicht zu präferieren, denn die Kursbildung auf Aktienmärkten ist notwendigerweise in einem ökonomischen Kontext zu sehen. Sie ist das Ergebnis einer ökonomischen Bewertung und wird zentral beeinflusst durch die Gewinnentwicklung der jeweils betrachteten Unternehmen.

Im Weiteren folgen wir der Vorgehensweise und der Analyse von Albrecht/Kantar (2003) und präsentieren in den Abschnitten 3 und 4 sowie in Anhang B einige der elementaren Ergebnisse dieser Arbeit ${ }^{34}$.

32 Da - wie in Abschnitt 2.4 ausgeführt - im Rahmen eines Random Walk kein entsprechender fairer Prognosewert existiert.

33 Die so gewählte Vorgehensweise knüpft an an die Ergebnisse in Albrecht (2001), der auf der Basis einer fundamentalen Analyse für den Deutschen Aktienmarkt zu dem Schluss kam, dass die Random WalkHypothese über längere Zeiträume nicht aufrechterhalten werden kann.

34 In der Ausarbeitung Albrecht/Kantar (2003) steht primär die statistische Analyse der KGV-Entwicklung des DAX im Vordergrund. Insbesondere werden eine Reihe von formalen statistischen Tests zur Überprïfung der Hypothesen "Random Walk versus Mean Reversion" durchgeführt. 


\section{Die Entwicklung des DAX-KGV}

Ausgangspunkt der weiteren Analyse ist die in der (kommerziellen) Datenbank Datastream enthaltene Zeitreihe der Kurs/Gewinn-Verhältnisse des DAX auf Monatsbasis. Um sowohl zu einem aktuellen als auch repräsentativen Sample zu kommen, legen wir den weiteren Analysen ausgehend von dem aktuellst verfügbaren KGV-Wert des Monats 06/2003 eine 30-jährige Historie zugrunde, d.h. die analysierte Datenreihe besteht aus den KGV-Werten von 07/1973 - 06/2003. Dies sind insgesamt 360 Werte. Sämtliche weitere statistische Auswertungen wurden mit dem Ökonometrieprogramm EViews 4.1 durchgeführt. Abbildung 1 zeigt zunächst die Entwicklung dieser Zeitreihe in einer graphischen Darstellung.

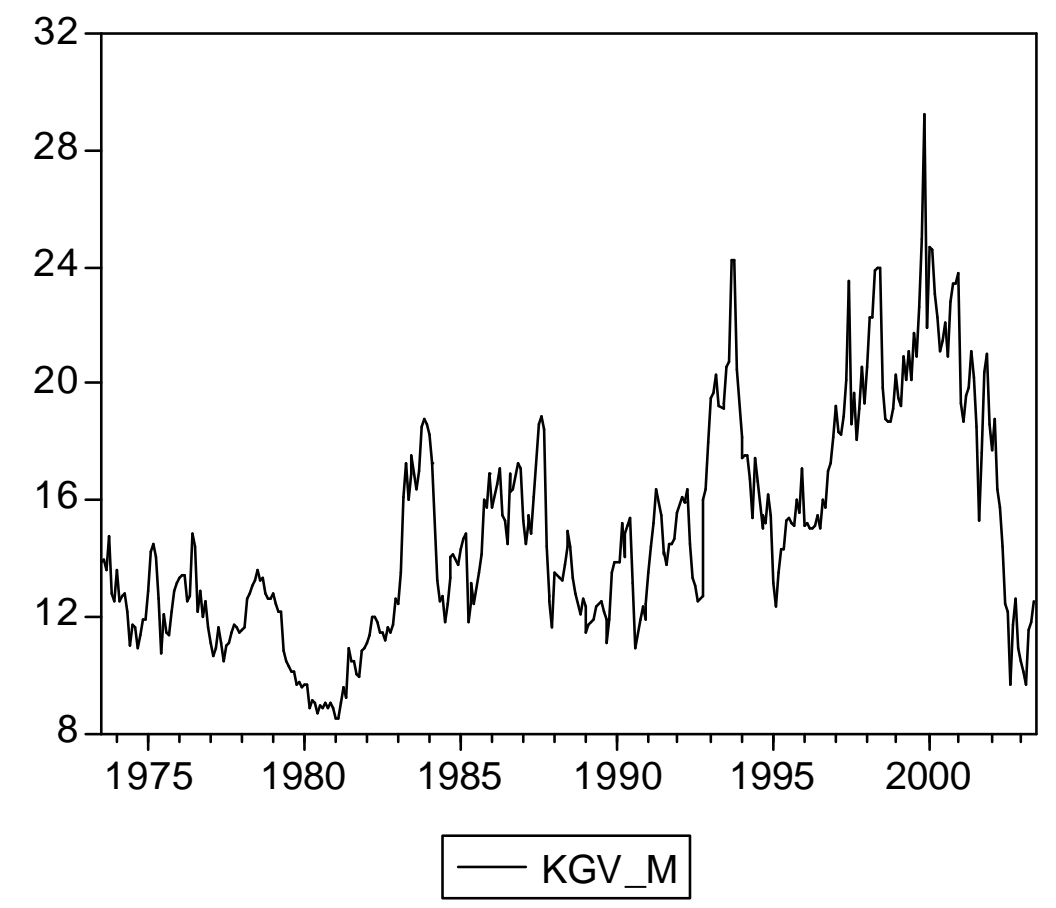

Abb. 1: Entwicklung des DAX-KGV von 07/73 - 06/03 auf monatlicher Basis (Quelle: Datastream)

Wie die graphische Darstellung deutlich macht, bewegt sich das KGV auf Monatsbasis weit überwiegend in einer Range von $10-20$. Werden diese Werte unter- oder überschritten, wird früher oder später eine Gegenreaktion ausgelöst, die die KGV-Werte zurück in diese Range treibt. Langfristige Unterschreitungen eines KGV-Wertes von 10 können ebenso wie längere Überschreitungen der Marke von 20 als Marktübertreibungen gewertet werden. Kurzfristige Unterschreitungen der Marke von 10 sind in den Monaten 10/81, 09/01 und 03/03 zu konstatieren, eine längere Unterschreitungsphase ist von 10/79 bis 05/81 zu verzeichnen. Der realisierte Tiefstwert ist 8.50 (dies geschah in den Monaten 01/81 und 02/81). Eher kurzfristige 
Überschreitungen der 20er Marke sind zu konstatieren in den Monaten 04/93, 08/93 - 12/93, $06 / 97+07 / 97,12 / 97,02 / 98-07 / 98,01 / 99,06 / 01+07 / 01$ sowie 11/01 + 12/01. Nur in der letzten Boomphase der Aktienmärkte ist eine länger anhaltende Überschreitung der Marke von 20 zu verzeichnen, konkret war dies die Periode 04/99 - 01/01. Der realisierte Maximalwert war dabei 29.20.

Die nachfolgende Tabelle enthält noch die Angaben über Mittelwert sowie ausgewählte Quantile $^{35}$ der DAX-KGV-Reihe.

\begin{tabular}{|c|c|}
\hline Mittelwert & 14.85 \\
Median & 14.15 \\
\hline 99\%-Quantil & 24.36 \\
95\%-Quantil & 22.20 \\
90\%-Quantil & 20.31 \\
\hline 10\%-Quantil & 10.80 \\
5\%-Quantil & 9.60 \\
1\%-Quantil & 8.80 \\
\hline
\end{tabular}

Tab. 1: Mittelwert und Quantile der bereinigten DAX-KGV-Reihe 07/73 - 06/03

Nimmt man das $10 \%$-Quantil ${ }^{36}$ bzw. das $90 \%$-Quantil ${ }^{37}$ als Maßstab für eine "normale" KGVRange, so deckt sich dies weitgehend mit der bereits gegebenen visuellen Einschätzung, dass dies die Zone zwischen 10 und 20 ist. Be i Verwendung des 5\%-bzw. 95\%-Quantils als Maßstab für eine normale KGV-Range wird diese Zone noch etwas ausgedehnt.

Schon eine erste Inspektion der empirischen Datenlage spricht damit durchaus für die Mean Reversion-These, denn es offenbart sich ein typisches Rückkehrverhalten in die KGV-Zone 10 - 20. Eine beliebige KGV-Steigerung bzw. beliebige KGV-Verminderung, wie sie bei einem Random Walk möglich wäre, ist nicht zu verzeichnen. Eine weitergehende explorative Analyse der DAX-KGV-Reihe ist in Anhang B.1 zu finden. Eine Analyse auf der Grundlage formaler statistischer Tests (Einheitswurzeltests, Stationaritätstests) wird in Albrecht/Kantar (2003) vorgenommen. Sämtliche Ergebnisse sprechen eindeutig und in einheitlicher Weise dafür, dass die Hypothese des AR(1)-Prozesses die analysierte Zeitreihe besser statistisch repräsentiert als der Random Walk. 
Damit ist nicht der aktuelle Wert (hier des KGV) der beste Prädiktor für den "fairen Wert", sondern das Langfristmittel des AR(1)-Prozesses. Den Konsequenzen hieraus soll im Folgenden nachgegangen werden.

\section{Eine DAX-Projektion}

Ansatzpunkt für eine DAX-Projektion ist zunächst die in Anhang B.2 identifizierte und verifizierte Regressionsgleichung (B.2) und der damit verbundene langfristige Gleichgewichtswert des AR(1)-Prozesses in Höhe eines KGV-Wertes von 14.8. Als weiterer Ausgangspunkt dienen die empirischen Verhältnisse per Ultimo 2002. Zu diesem Zeitpunkt ist ein DAXStand von $2892.63 \mathrm{zu}$ verzeichnen, das zugehörige DAX-KGV weist einen Wert von 10.90 auf. Gemessen am 10\%-Quantil in Höhe von 10.8 der KGV-Reihe, vgl. Tabelle 1, war damit der DAX praktisch an der Grenze zu einer systematischen Unterbewertung. Gegeben diese Ausgangssituation beträgt die rechnerische Gewinngröße pro DAX-KGV-Punkt 265.38. Unter Anwendung des langfristigen Gleichgewichtswertes für die DAX-KGV-Reihe in Höhe von $m=14.80$, ergibt sich daraus ein entsprechender DAX-Stand von 3927.60. Dies beinhaltet zunächst eine DAX-Prognose bei Anwendung des Gleichgewichts-KGVs und bei unveränderter Gewinnsituation. ${ }^{38}$ Eine DAX-Projektion unter Berücksichtigung des künftigen $G e$ winnwachstums erhält man des Weiteren auf die in Anhang $\mathrm{C}$ formal dargestellte Weise.

Der projizierte DAX-Stand hängt dabei ab vom KGV-Wachstum, vom Gewinnwachstum und vom angenommenen Startwert des DAX. Ausgangskurs und Ausgangs-KGV sind bekannt. Das KGV-Wachstum ergibt sich als das Verhältnis des Gleichgewichts-KGVs in Höhe von $14.80 \mathrm{zu}$ dem Ausgangs-KGV. Offen ist damit nur noch die Substantiierung der Gewinnwachstumsrate. Diese kann man aus den entsprechenden Werten der Datastream-Datenbank für die Entwicklung des DAX sowie des DAX-KGVs berechnen. Für die Jahre 1974-2002 ergibt sich dabei ein durchschnittliches jährliches Gewinnwachstum von $6.46 \%$.

Unter Ansatz dieses mittleren Wertes sowie der sonstigen bereits spezifizierten Annahmen ergibt sich auf der Basis der Beziehung (C.2) des Anhangs C ein prognostizierter DAX-Stand von 4181.30. Diese Vorgehensweise nimmt dabei eine einjährige Sichtweise ein, d.h. unterstellt, dass das mittlere DAX-Wachstum für eine Projektionsperiode von einem Jahr wirksam 
ist. Entsprechend kann man vorgehen, wenn man einen mehrjährigen Projektionszeitraum ansetzt und unterstellt, dass das angenommene mittlere DAX-Gewinnwachstum in Höhe von 6.46\% über den gesamten Projektionszeitraum gültig ist. Die Wahl eines mehrperiodigen Projektionszeitraums ist auch deswegen plausibler, weil dann das KGV entsprechende Zeit bekommt, sich auf sein Langfristmittel hin zu bewegen. Unterstellt man einen Projektionszeitraum von 3 Jahren, so führt dies zu einem DAX-Stand von 4739.00, bei einem Projektionszeitraum von 5 Jahren führt dies zu einem Wert von 5371.10. Unterstellt wird dabei jeweils eine Bewertung der Gewinnsituation zum Gleichgewichts-KGV von 14.8. Die Tabelle 2 fasst die vorstehend gewonnenen Ergebnisse zusammen.

\begin{tabular}{|c|c|c|}
\hline \multicolumn{3}{|l|}{ DAX-Stand bei } \\
\hline (a) unveränderter Gewinnsituation (bzw. Gewi & achstum von null) & 3927.60 \\
\hline (b) mittlerem Gewinnwachstum von $6.46 \%$ fü & $\begin{array}{l}\text { 1 Jahr } \\
\text { 3 Jahre } \\
5 \text { Jahre }\end{array}$ & $\begin{array}{l}4181.30 \\
4739.00 \\
5371.10\end{array}$ \\
\hline
\end{tabular}

Tab. 2: $\quad$ Projizierte DAX-Stände bei Annahme eines Gleichgewichts-KGV von 14.8.

\section{Langfristcharakter des Versicherungsgeschäfts und Bewertung}

Das zentrale Ergebnis des Abschnitts 3 besteht darin, dass die Hypothese des AR(1)Prozesses die analysierte Zeitreihe (hier: DAX-KGV) besser statistisch repräsentiert als der Random Walk. Dies ist eine weitere Bestätigung und Ergänzung der in der wissenschaftlichen Literatur $^{39} \mathrm{zu}$ findenden Evidenz, dass an Aktienmärkten über längere Zeiträume Mean Reversion-Effekte bestehen. Damit ist nicht mehr der aktuelle Aktienkurs bzw. der aktuelle Wert eines Aktienportfolios der beste Prädiktor für eine faire Bewertung, sondern das aus der statistischen Repräsentierung der Daten (im Falle des DAX-KGVs: AR(1)-Prozess) resultierende Langfristmittel. Geht man von einem prospektiven Verständnis der Informationsfunktion der Rechungslegung aus, so entspricht dieses Langfristmittel der bestmöglichen Einschätzung des "inneren" Wertes des analysierten Aktienportfolios.

Allerdings ist anzumerken, dass die zentrale Mean Reversion-Eigenschaft, dass temporäre Abweichungen vom Langfristmittel früher oder später korrigiert werden, keine kausale Cesetzmäßigkeit ist, sondern eine statistische. Die Dauer der Bereinigung einer solchen temporä- 
ren Abweichung ist daher nicht exakt bestimmbar, sondern zufallsabhängig. Entsprechend können Bewertungsübertreibungen oder Bewertungsuntertreibungen auf Aktienmärkten auch längere Zeit bestehen bleiben. Insofern kommt es bei der Verwendung eines solchen Bewertungsmaßstabes entscheidend auf die Fähigkeit an, die Aktienanlage auch während der Dauer einer solchen Bereinigungsphase von Bewertungsuntertreibungen „durchzuhalten“, d.h. nicht vorzeitig zu Marktwerten liquidieren zu müssen. Gerade bei Lebensversicherungsunternehmen, allgemeiner bei Unternehmen der Personenversicherung, ist diese Fähigkeit aufgrund ihrer spezifischen Geschäftstätigkeit, insbesondere der spezifischen Langfristigkeit der abgeschlossenen Versicherungsverträge, aber in substantieller Hinsicht gegeben. Auf diesen Gesichtspunkt soll im Folgenden noch näher eingegangen werden.

Dabei ist - wie bereits in Abschnitt 2.2 ausgeführt - zum Zwecke einer bilanziellen Bewertung $^{40}$ grundsätzlich von dem Going Concern-Prinzip auszugehen, d.h. von dem Einsatz der Aktiva im normalen Leistungsprozess des Unternehmens.

Unterstellen wir dabei, dass das Neugeschäft nicht dazu herangezogen werden soll, mögliche strukturelle Probleme (auf der Aktivseite) des Bestandes zu kompensieren, so ist zur Abschätzung der Notwendigkeiten einer Auflösung der Aktienposition konsequenterweise hypothetisch von dem Fall eines Lebensversicherers auszugehen, der für einen Neuzugang geschlossen und dessen Bestand synchron zu der vertragsgemäßen Fälligkeit der Versicherungsleistungen "abgewickelt" wird. Die damit einhergehende Abwicklung der Aktiva wird naturgemäß sehr stark bestimmt sein von der Bestandsstruktur, insbesondere der Laufzeitenstruktur der bestehenden Versicherungsverträge. Gehen wir - primär zu Illustrationszwecken - aus von einem Bestand mit einer (durchaus konservativ angesetzten) mittleren Restlaufzeit von 15 Jahren und nehmen ferner an, dass die Versicherungsleistungen über diesen Zeitraum in gleichmäßiger Weise fällig werden. Gehen wir des Weiteren vereinfachend aus von einem Kapitalanlageportfolio, das aus 80\% Zinstiteln und 20\% Aktien besteht. Löst man zunächst den Bestand an Festzinstiteln auf und geht erst danach an die Liquidierung der Aktienpositi-

40 Aber auch aus Sicht einer Liquiditätsplanung können in der Lebensversicherung bzw. allgemeiner der Personenversicherung aufgrund der Stabilität der biometrischen Rechnungsgrundlagen und der damit einhergehenden guten Prognostizierbarkeit der Zeitpunkte sowie der Höhen der Versicherungsleistungen die in den nächsten Perioden benötigten liquiden Mittel sehr stabil ermittelt und im Rahmen eines Asset/LiabilityMatching durch entsprechende gut liquidierbare Assets bedeckt werden. Nimmt man noch das Faktum der Vorauszahlung der Prämien hinzu, so erkennt man, dass auch aus Sicht der Liquiditätspolitik Notwendigkeiten einer kurz- oder mittelfristigen Liquidierung von Aktiva nur schwach ausgeprägt sind. Hinzu kommt, dass selbst dann zunächst die anderen Assetpositionen aufgelöst werden können, die vom Umfang her die Aktienposition eines Lebensversicherers ja bei weitem dominieren. 
on, so ist evident, dass rein rechnerisch zwölf Jahre vergehen, bevor die Notwendigkeit zur graduellen Liquidierung von Aktien besteht. Wenn auch stark vereinfachend und im konkreten Anwendungsfall im Hinblick auf die spezifische Ablauf- und Assetstruktur des zu analysierenden Versicherungsunternehmens zu modifizieren, so macht dieses Beispiel doch sehr deutlich, dass aufgrund des ausgeprägten Langfristcharakters des Lebens- bzw. allgemeiner des Personenversicherungsgeschäfts ${ }^{41}$, eine Notwendigkeit einer vor- oder frühzeitigen Auflösung der Aktienposition nicht besteht, sondern das Gegenteil der Fall ist. Damit lässt sich die aus modelltheoretischer Sicht optimale Vorgehensweise einer bilanziellen Bewertung ${ }^{42}$ der Aktienposition zum fairen langfristigen, etwa wie in Abschnitt 4 bestimmten, Wert geradezu idealtypisch im Rahmen des konkreten Anwendungsfalls der Lebensversicherung realisieren und es schließt sich damit der Kreis der Argumente.

Abschließend soll noch darauf hingewiesen werden, dass der in der vorliegenden Arbeit herausgearbeitete Grundgedanke der Notwendigkeit einer längerfristigen Perspektive für die Bewertung einer Aktienanlage im Zusammenhang mit Aktivitäten der Lebensversicherung oder allgemeiner der Personenversicherung auch im Rahmen der internationalen Rechnungslegung durchaus nicht unbekannt ist. Konkretes Beispiel ist das Statement of Financial Accounting Standards Nr. 87 hinsichtlich des "Employers' Accounting for Pensions", mithin den Rechnungslegungsvorschriften nach dem Regelwerk des Financial Accounting Standards Boards (FASB) im Hinblick auf betriebliche Pensionsverpflichtungen (die nach internationalen Cepflogenheiten typischerweise in betriebliche Pensionsfonds ausgelagert sind).

In Par. 30 der FAS 87 wird im Hinblick auf die Bewertung der Kapitalanlagen des betrieblichen Pensionsplans ausgeführt:

\footnotetext{
"The expected return on plan assets shall be determined based on the expected long-term rate of return on plan assets and the market-related value of plan assets. The market-related value of plan assets shall be either fair value or a calculated value that recognizes changes in fair value in a systematic and rational manner over not more than five years. Different ways of calculating market-related value may be used for different classes of assets (for example, an employer might use fair value for bonds and a five-year-moving-average value for equities), but the manner of determining market-related value shall be applied consistently from year to year for each asset class.
}

41 So enthalten etwa auch die Erwägungsgründe zur EU -Pensionsfonds-Richtlinie unter Punkt (33) die explizite Feststellung, dass Einrichtungen der betrieblichen Altersvorsorge sehr langfristige Investoren mit geringen Liquiditätsrisiken und daher sogar in der Lage sind, in nicht liquide Vermögenswerte zu investieren. Zum genauen Wortlaut vgl. etwa die Wiedergabe der EU-Pensionsfonds-Richtlinie in Betriebliche Altersversorgung 3/2003, S. 250.

42 Dies schließt keinesfalls aus, dass auch der Zeitwert der Aktienposition eines Lebensversicherers im Rahmen der Informations- und Offenlegungspflichten des Jahresabschlusses darzulegen ist. Dies würde jedoch im Anhang geschehen. 
Es besteht mithin die Möglichkeit des Ansatzes eines Market Related-Values, wobei dieser auf den Marktwerten der jeweils letzten (maximal) 5 Jahre beruht, beispielsweise auf dem entsprechenden gleitenden Durchschnittswert. Wenn dieser Ansatz auch eher als pragmatisch zu beurteilen ist und nicht auf modelltheoretischen Erwägungen beruht, wie der in dieser Arbeit favorisierte Ansatz des Langfristmittels des zugrundeliegenden AR(1)-Prozesses, so weist er doch in die richtige Richtung und anerkennt, dass der Marktwert im Zusammenhang mit langfristigen Aktivitäten (hier: Pensionsverpflichtungen) nicht notwendigerweise der angemessene bilanzielle Wert ist.

In Par.175 der FAS 87 wird zu dem Aspekt der Volatilität ausgeführt:

"... in the case of pension liabilities, volatility may not be entirely a faithful representation of changes in the status of the obligation ... . It may also reflect an unavoidable inability to predict accurately the future events that are anticipated in making period-to-period measurements".

Das FSAB weist somit explizit auf die Probleme hin, die eine periodische Rechnungslegung im Zusammenhang mit langfristigen Aktivitäten induziert und negiert, dass die hierdurch induzierte Volatilität relevante Rechnungslegungsinformationen beinhaltet.

Die Regelungen der FAS 87 sind umso bemerkenswerter, als dass die zu bilanzierende Langfristaktivität (Pensionsverpflichtungen) hier nicht - wie im Falle von Lebensversicherungsoder Personenversicherungsunternehmen - das Kerngeschäft der betreffenden Unternehmen darstellt, sondern nur ein Aktivitätssegment. Offenbar ist also allein die Langfristigkeit der zu bilanzierenden Aktivität ausschlaggebend für die Verwerfung des Markwertes als angemessenem Bilanzwert.

\section{Zusammenfassung und Schlussfolgerungen}

Gegenstand der vorliegenden Ausarbeitung ist die Frage nach der Bestimmung eines angemessenen Wertmaßstabes aur Einschätzung des "fairen Wertes" des Aktienportfolios eines Lebensversicherungsunternehmens aus ökonomischer und statistischer Perspektive. Der zu bestimmende "faire Wert" soll dabei bestimmten grundlegenden Anforderungen genügen, der Anforderung der Modellgestütztheit, der Anforderung der statistischen Repräsentanz sowie der Anforderung der bestmöglichen Werteinschätzung. Ausgangspunkt ist dabei das Going Concern-Prinzip, d.h. es wird - in Einklang mit den Rechnungslegungsgrundsätzen nach HGB und IAS - die Prämisse der Fortsetzung der Unternehmenstätigkeit gesetzt und die Er- 
mittlung eines fairen Wertes des Aktienportfolios eines Lebensversicherungsunternehmens erfolgt im Rahmen der normalen Unternehmensaktivitäten.

Es wird sodann dargelegt, dass die drei finanzmathematischen Standardmodelle Martingal, Random Walk und AR(1)Prozess - letzteres als Basismodell für die Erfassung von Mean Reversion-Effekten - grundsätzlich alle gestellten Anforderungen erfüllen und der modellgestützte faire Wert jeweils dem besten Prädiktor (im Sinne der statistischen Theorie) des künftigen Wertes, gegeben die aktuell vorhandenen Informationen, entspricht. Damit ist der Wertmaßstab (bester Prädiktor) zur angestrebten Ermittlung eines fairen Wertes gefunden und es muss zu seiner konkreten Spezifikation nur noch geklärt werden, welches der finanzmathematischen Alternativmodelle die Daten am besten repräsentiert.

Dabei stellt sich heraus, dass die Martingal-Hypothese weder aus modelltheoretischer noch aus empirischer Sicht haltbar ist. Nur im Rahmen der Martingal-Hypothese erfüllt aber der Marktwert die Eigenschaft des besten Prädiktors, d.h. nur in diesem Falle wäre er auch unter der dargelegten ökonomischen-statistischen Perspektive als fairer Wert anzusehen. Dies zeigt zugleich, dass das Verständnis des Marktwertes als Fair Value gemäß der nationalen und internationalen Rechnungslegungsnormen nicht notwendigerweise ein Fair Value im Sinne eines ökonomischen Gleichgewichtsansatzes ist, sondern sich das Attribut fair primär auf die Objektivierbarkeit des beizulegenden Wertes (Orientierung an Markttransaktionen und nicht an der individuellen Einschätzung) bezieht. Dies ist aber ein fundamentaler Unterschied und relativiert damit die Begründungsbasis für die (internationale) Vorherrschaft des Fair ValueParadigmas erheblich. Nicht zuletzt der dramatische Kursverfall der Aktienmärkte in den Jahren 2000 - 2002 bestätigt eindrucksvoll, dass die Marktwerte z.B. des Jahres 1999 keinesfalls faire Widerspiegelungen des wahren Wertes der zugrundeliegenden Aktien gewesen sind, sondern im Gegenteil eher erhebliche Informationsverzerrungen beinhaltet haben.

Da die Martingal-Hypothese weder theoretisch noch empirisch haltbar ist, spitzt sich die Frage nach der Spezifikation eines fairen Wertes, zu dem ein Aktienportfolio bewertet werden kann, somit zu auf die Frage "Random Walk oder Mean Reversion?" Zur Klärung dieser Frage existieren eine Reihe von einschlägigen wissenschaftlichen Untersuchungen. Dabei wird im Rahmen der vorliegenden Ausarbeitung der Vorgehensweise von Albrecht/Kantar (2003) gefolgt, die Analyse nicht - wie bislang üblich - auf der reinen Kurs- bzw. Renditeebene durchzuführen, sondern auf der Fundamentalebene in Termen der Entwicklung des Kurs/Gewinn-Verhältnisses (hier: des DAX). Da die Kursbildung auf Aktienmärkten notwendigerweise das Ergebnis einer ökonomischen Bewertung ist und diese zentral durch die $\mathrm{Ce}$ - 
winnentwicklung der jeweils betrachteten Unternehmen bestimmt wird, ist dieser Ansatzpunkt zu präferieren.

Sämtliche Ergebnisse der entsprechend durchgeführten statistischen Analysen sprechen eindeutig und in einheitlicher Weise dafür, dass die Hypothese eines AR(1)-Prozesses die analysierte Zeitreihe besser statistisch repräsentiert als der Random Walk. Damit ist nicht der aktuelle Wert (hier das KGV) der beste Prädiktor für den "fairen" Wert, sondern das Langfris tmittel des AR(1)-Prozesses. Dieses wird ermittelt und nimmt einen Wert in Höhe von 14.8 an.

Ausgehend von dem solchermaßen ermittelten Gleichgewichts-DAX-KGV können sodann auf der Basis unterschiedlicher Annahmen über das Gewinnwachstum und den Prognosehorizont - korrespondierende Projektionen für den künftigen DAX-Stand vorgenommen werden. Diese Projektionen stellen im Rahmen der Zielsetzung der Ermittlung eines ökonomischstatistisch gestützten fairen Wertes eines Aktienportfolios (hier repräsentiert durch den DAX) eines Lebensversicherungsunternehmens die angestrebte Substantiierung eines solchen fairen Wertes dar.

Abschließend wird begründet, dass die aus modelltheoretischer Sicht optimale Vorgehensweise einer bilanziellen Bewertung der Aktienposition zum fairen langfristigen Wert sich geradezu idealtypisch im Rahmen des konkreten Anwendungsfalls der Lebensversicherung - bzw. allgemeiner der Personenversicherung - realisieren lässt. Aufgrund des ausgeprägten Langfristcharakters des Lebensversicherungsgeschäfts, begründet durch die spezifische Langfristigkeit der bestehenden Versicherungsverhältnisse, bestehen keine Notwendigkeiten zu einer vor- oder frühzeitigen Auflösung der bestehenden Aktienposition. Im Gegenteil, im Rahmen eines normalen Geschäftsbetriebs besteht eine nachhaltige Fähigkeit der Lebensversicherer, auch sehr lange Bereinigungsphasen im Sinne von Bewertungsuntertreibungen auf den Aktienmärkten durchzustehen, ohne dabei das Aktienengagement zu Marktwerten liquidieren zu müssen. Es wird in diesem Zusammenhang darauf hingewiesen, dass auch im Rahmen internationaler Rechnungslegungsstandards Beispiele zu finden sind, bei Aktivitäten mit typischem Langfristcharakter nicht vom Marktwert als angemessenem bilanziellen Wert auszugehen. Exemplifiziert wird dies am Beispiel der FAS 87, die das Employers' Accounting for Pensions behandelt. 


\section{Anhang A: Modellgrundlagen}

\section{A.1 Beste Prädiktion}

Gehen wir aus von einer (zufallsabhängigen) Wertentwicklung $V_{0}, V_{1}, \ldots, V_{t}$, die wir bis zum Zeitpunkt t beobachtet haben. Basisziel einer Prädiktion (auch Prognose oder Projektion genannt) der Wertentwicklung ist die Bestimmung eines Prognosewertes $V_{t+s}^{*}$ zu einem zukünftigen Zeitpunkt $t+s(s \geq 1)$ gegeben die Information der bisher beobachteten Werte, d.h. allgemein

$$
V_{t+s}^{*}=F\left(V_{t}, \ldots, V_{0}\right)
$$

Es lässt sich nun zeigen ${ }^{43}$, dass der bedingte Erwartungswert ${ }^{44} E\left(V_{t+s} \mid V_{t}, \ldots, V_{0}\right)$ unter allen Funktionen F (die gewisse Regularitätsanforderungen erfüllen) einen minimalen quadratischen Prognosefehler ${ }^{45}$ (Mean Square Error, MSE) aufweist, d.h.

$$
\begin{aligned}
& E\left\{\left[V_{t+s}-E\left(V_{t+s} \mid V_{t}, \ldots, V_{0}\right)\right]^{2}\right\} \\
& \leq E\left\{\left[V_{t+s}-F\left(V_{t}, \ldots, V_{0}\right)\right]^{2}\right\} \text { für "alle" } \mathrm{F} .
\end{aligned}
$$

Unter dem MSE-Kriterium ist damit

$$
V_{t+s}^{*}:=E\left(V_{t+s} \mid V_{t}, \ldots, V_{0}\right)
$$

der bestmögliche Prognosewert bzw. die beste Prädiktion (auch: bester Prädiktor) für die Realisation des künftigen Wertes der betrachteten Entwicklung, wenn in die Prognose lediglich die Information der bereits beobachteten Werte $V_{0}, V_{1}, \ldots, V_{t}$ eingehen darf ${ }^{46}$.

43 Vgl. im Zeitreihenkontext etwa Granger/Newbold (1977, S. 115 ff.), Hamilton (1994, S. 72 f.).

44 Zum bedingten Erwartungswert vgl. etwa Albrecht/Maurer (2002, S. 161 f.).

45 Wie Granger (1969) nachweist, bleibt diese Eigenschaft des bedingten Erwartungswertes auch bei allgemeineren symmetrischen Verlustfunktionen erhalten.

46 Auch für allgemeinere Informationsmengen existiert ein analoges Resultat in Termen des bedingten Erwartungswertes. 


\section{A.2 Martingal-Hypothese}

Die zufallsabhängige Entwicklung $\left\{V_{0}, V_{1}, \ldots, V_{t}, \ldots\right\}$ der interessierenden Variablen wird als (zeitdiskretes) Martingal ${ }^{47}$ bezeichnet, wenn gilt

$$
\mathrm{E}\left(V_{t+1} \mid V_{t}=v_{t}, V_{t-1}=v_{t-1}, \ldots, V_{1}=v_{1}, V_{0}=v_{0}\right)=v_{t}
$$

und dies für $t \geq 0$ für alle Verläufe ("Geschichten") $\left\{v_{0}, v_{1}, \ldots, v_{t}, \ldots\right\}$ von Entwicklungen gültig ist.

Die Martingaleigenschaft besagt mithin, dass der erwartete Wert der Variablen am Ende der nächsten Periode, gegeben die Information $V_{t}=v_{t}$ über den "heutigen" Wert sowie über die "vergangene" Wertentwicklung $V_{t-1}=v_{t-1}, \ldots, V_{0}=v_{0}$, stets dem aktuellen Wert entspricht. Auch für die künftigen Werte zu Zeitpunkten $T>t+1$ ändert sich die Sit uation nicht, es gilt:

$$
\mathrm{E}\left(V_{T} \mid V_{t}, \ldots, V_{0}\right)=V_{t}
$$

Martingale können als modelltheoretische Umsetzung der Konzeption eines so genannten fairen Spiels interpretiert werden. Repräsentiert $V_{t}$ den Vermögensstand (kumulierte Gewinne und Verluste) aus der Teilnahme an einem derartigen Spiel, so ist der erwartete Vermögensstand nach der nächsten Spielperiode gerade der heutige Vermögensstand bzw. der erwartete Vermögenszuwachs gleich null. Dies schließt nicht aus, dass sich im konkreten Spielverlauf auch erhebliche positive oder negative Vermögensänderungen realisieren können. Das Spiel ist aber nicht als systematische "Gewinn- bzw. Verlustmaschine" konzipiert.

Repräsentiert $\left\{V_{t}\right\}$ den Verlauf der Kursentwicklung eines Finanztitels, so besagt die Martingal-Hypothese, dass der erwartete Kurszuwachs gleich null ist. Positive und negative Kursänderungen halten sich wahrscheinlichkeitsgewichtet die Waage. Aus einer Prognoseperspektive impliziert daher die MartingalHypothese, dass der heutige Wert der beste (MSE-)Prädiktor $^{48}$ für den Wert am Ende der nächsten Periode ist.

Gilt für die Wertentwicklung die Eigenschaft

$$
E\left(V_{t+1} \mid V_{t}, \ldots, V_{0}\right) \geq V_{t}
$$

\footnotetext{
47 Vgl. hierzu allgemein Albrecht/Maurer (2002, S. 138 f.).

48 Im Sinne der Ausführungen in Anhang A.1.
} 
d.h. gilt in (A.4) - und dann ebenso in (A.5) - anstelle des Gleichheitszeichens ein Größeroder Gleich-Zeichen, dann spricht man von einem Submartingal. In diesem Falle ist der erwartete künftige Wert somit immer höher als der heutige Wert.

\section{A.3 Random Walk-Hypothese}

Bezeichnet wiederum $V_{t}$ die (diskrete) zeitliche Entwicklung der interessierenden Variablen, gemessen zu den Zeitpunkten $t=0,1,2, \ldots$, so besitzt das Grundmodell ${ }^{49}$ eines (arithmetischen) Random Walk mit Drift die Charakterisierung $(t=1,2,3, \ldots)$

$$
\Delta V_{t}:=V_{t}-V_{t-1}=m+Z_{t}
$$

wobei weiterhin gilt:

$$
E\left(Z_{t}\right)=0, \quad \operatorname{Var}\left(Z_{t}\right)=\sigma^{2}, \quad \operatorname{Cov}\left(Z_{t}, Z_{t-1}\right)=0
$$

Die absoluten Wertveränderungen ${ }^{50}$ (Zuwächse) über eine Periode (Monat, Jahr) lassen sich somit durch eine zeitlich konstante Komponente, quantifiziert durch den sog. Driftparameter $m$, beschreiben, die durch einen Zufallsprozess $\left\{Z_{t}\right\}$ überlagert wird. Die Forderung (A.7b) besagt, dass diese zufälligen Überlagerungen einen sog. White Noise-Prozess darstellen. Die Zufallsgrößen $Z_{t}(t \geq 1)$ sind unkorreliert und identisch verteilt mit einem Erwartungswert von null und einer in der Zeit konstanten Varianz.

In expliziter Form besitzt der Random Walk die Darstellung

$$
V_{t}=v_{0}+m t+Z_{1}+\ldots+Z_{t}
$$

Die zufällige Abweichung von dem Grunde nach linearen Trend entsteht somit im Zeitablauf durch eine Akkumulation der einzelnen Fehler (Schocks). Diese mögliche "Aufschaukelung" der Fehler führt dazu, dass die Abweichungen vom zugrunde liegenden Trend dem Grunde

Vgl. hierzu etwa Albrecht/Maurer (2002, S. 140 ff.).

50

Bei der Anwendung dieser Modellbildung auf Aktienkurse entspricht dabei $V_{t}$ standardmäßig den logarithmierten Aktienkursen bzw. $\Delta V_{t}$ der zeitstetigen Periodenrendite. Äquivalent hierzu ist das Modell eines multiplikativen Random Walks, vgl. etwa Albrecht/Maurer (2002, S. 141). 
nach "beliebig groß" werden können. ${ }^{51}$ Insofern ist die erwartete Entwicklung $E\left(V_{t}\right)=v_{0}+m t$ keine zuverlässige Prognose für die sich künftig realisierende Entwicklung. Das Random Walk-Modell beinhaltet daher im Kern eine Nicht-Prognostizierbarkeit der

\section{künftigen Wertveränderungen}

In alternativer Form wird dies ausgedrückt ${ }^{52}$ durch die Angabe des besten (MSE-)Prädiktors $^{53}$. Es gilt beim Random Walk

$$
E\left(V_{t+s} \mid V_{t}, V_{t-1}, \ldots, V_{0}\right)=v_{0}+m(t+s)+\sum_{i=1}^{t} Z_{i}
$$

Jeder vergangene Schock hat damit Auswirkungen auf alle zukünftigen Werte. Aufgrund dieser Eigenschaft der Fehlerakkumulation bezeichnet man den Random Walk auch als integrierten Prozess. ${ }^{54}$ Die Form der zufälligen Überlagerungen der Gesamtentwicklung $V_{t}$ des Prozesses ist beim Random Walk nicht stationär, da die Überlagerung $Z_{1}+\ldots+Z_{t}$, instationär (in den Parametern) ist. Durch Differenzenbildung, d.h. durch den Übergang zu den Zuwächsen gemäß (A.7a) wird der Random Walk allerdings stationär. Man bezeichnet den Random Walk daher auch als differenzenstationären Prozess bzw. als integriert von der Ordnung 1.

\section{A.4 Mean Reversion-Hypothese}

Das Basismodell zur Modellierung von Mean Reversion-Effekten ist der autoregressive Prozess der Ordnung 1, kurz AR(1)-Prozess. Die Wertentwicklung eines AR(1)-Prozesses besitzt dabei die folgende Charakterisierung ${ }^{55}(t=1,2,3, \ldots ;|a|<1)$ :

$$
V_{t}-m=a\left(V_{t-1}-m\right)+Z_{t}
$$

wobei der Überlagerungsprozess $Z_{t}$ wiederum den Anforderungen gemäß (A.7b) genügt. Unter der Bedingung $|a|<1$ ist der AR(1)-Prozess ein stationärer Prozess.

Und zwar in systematischer Hinsicht, nicht nur im Hinblick auf die Stochastik.

Vgl. hierzu Franke et al. (2001, S. 171).

Im Sinne der Ausführungen in Anhang A.1.

Vgl. ebenda.

Vgl. etwa Buscher (2002, S. 204 f.) oder Gujarati (1995, S. 736 f.). 
Die Bedingungsgleichung (A.10a) für den AR(1)-Prozess impliziert, dass der Parameter $m$ das langfristige Mittel des Prozesses darstellt und der Wertverlauf dieses Prozesses bei temporären Abweichungen auf dieses Langfristmittel hingezogen wird. Je größer der Parameter $a$, desto stärker ist die Elastizität, mit der der Prozess zum Langfristmittel hingezogen wird. Für den Erwartungswert des Prozesses gilt ${ }^{56}$ :

$$
E\left(V_{t}\right)=m
$$

In der Anwendung geht man dabei davon aus, dass der beobachtete Prozess schon "lange läuft" bzw. eingeschwungen ist.

Der Parameter $a$ ist zudem identisch mit dem Autokorrelationskoeffizienten 1. Ordnung des AR(1)-Prozesses, d.h. $\rho\left(V_{t+1}, V_{t}\right)=a$.

Äquivalent zur Darstellung (A.10a) ist die folgende Charakterisierung eines AR(1)-Prozesses, die ebenfalls häufig in der Literatur zu finden ist ${ }^{57}$ :

$$
V_{t}=c+a V_{t-1}+Z_{t}
$$

Dies erkennt man, wenn man (A.10a) explizit nach $V_{t}$ auflöst und dann $c=m(1-a)$ setzt. Die Charakterisierung (A.10b) zeigt im Vergleich mit der Darstellung 1, dass der Random Walk ein Grenzfall des AR(1)-Prozesses für $a \rightarrow 1$ ist. Tests der Hypothese $H_{0}: a=1$ gegen $H_{1}: a<1$, sog. Einheitswurzel oder Unit Root-Tests, bilden entsprechend ein zentrales Instrumentarium zur Überprüfung der Kernfrage "Random Walk oder Mean Reversion?" der vorliegenden Ausarbeitung ${ }^{58}$. Zugleich wird deutlich, dass die Charakterisierung (A.10b) als Regressionsgleichung mit der erklärten Variablen $V_{t}$ sowie der erklärenden Variablen $V_{t-1}$ interpretiert werden kann. Die Durchführung der entsprechenden Regression führt zu einer Bestimmung der Parameter $c$ bzw. $a$ und dient als Ausgangspunkt vieler Einheitswurzeltests.

Die Existenz des Langfristmittels $m$, zu dem der AR(1)-Prozess "hingezogen" wird, beinhaltet zugleich den zentralen Unterschied zum Random Walk, bei dem es - wie bereits ausgeführt -

56 Vgl. etwa Buscher (2002, S. 204).

57 Vgl. etwa Franke et al. (2001, S. 146 f.) oder Hamilton (1994, S. 48).

58 Zur Durchführung von Einheitswurzeltests im Zusammenhang mit der vorliegenden DAX-KGV-Reihe vgl. Albrecht/Kantar(2003). 
(systematisch) zu beliebig großen Abweichungen vom zugrunde liegenden Trend kommen kann. In Termen der besten (MSE-)Prädiktion ${ }^{59}$ gilt $^{60,61}$ :

$$
E\left(V_{t+s} \mid V_{t}, V_{t-1}, \ldots, V_{0}\right)=m+a^{s}\left(V_{t}-m\right) .
$$

Der Einfluss der momentanen Abweichung $V_{t}-m$ des aktuellen Prozesswertes vom Langfristmittel nimmt somit mit geometrischer Rate ab, und insgesamt nähert sich die (beste) Prognose immer mehr dem Langfristmittel an.

59 Im Sinne der Ausführungen in Anhang A.1.

60 Vgl. etwa Hamilton (1994, S. 80).

61 Die Prognosegleichung (A.12) ist dabei ein Spezialfall der Wiener-Kolmogorov-Prognose, vgl. ebenda. 


\section{Anhang B: Statistische Analyse der DAX-KGV-Zeitreihe}

\section{B.1 Weitergehende explorative Analyse}

Kommen wir nunmehr zu weitergehenden Elementen einer explorativen Datenanalyse, der Analyse des Korrelogramms. Das Korrelogramm enthält die Autokorrelationen ${ }^{62}$ des Prozesses, d.h. die Korrelationen zwischen den zeitlich versetzten Gliedern der Zeitreihe. Abbildung B.1 enthält das in EViews 4.1 erstellte Korrelogramm.

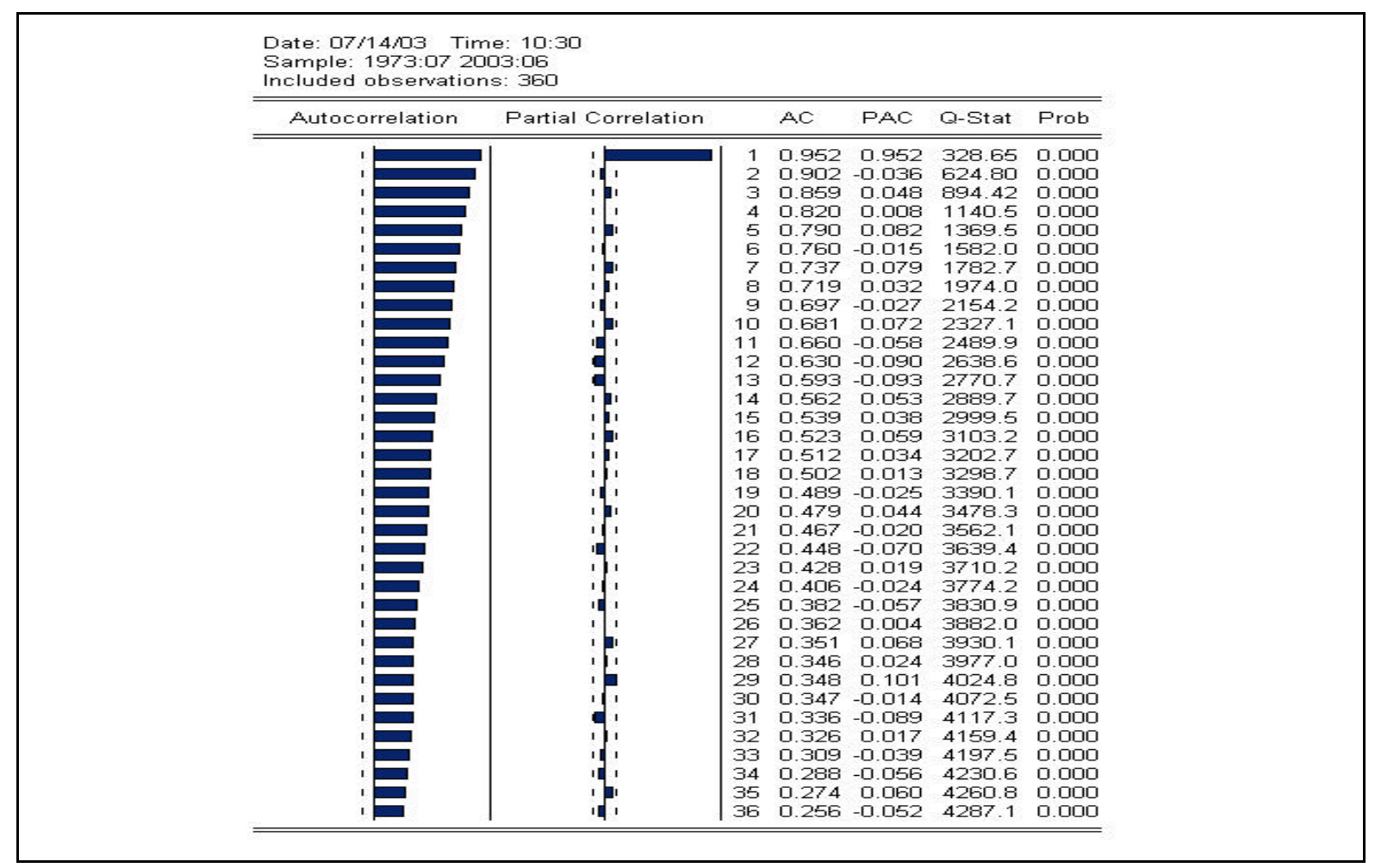

Abbildung B.1: Korrelogramm der bereinigten DAX-KGV-Zeitreihe 07/73 - 06/03

Hinsichtlich des Verlaufs der Autokorrelationen in Abhängigkeit von der zeitlichen Ordnung zeigt sich das typische ${ }^{63}$ monoton abklingende Verhalten eines AR(1)-Prozesses mit einem positiven Parameter $a$. Die Autokorrelationen sind dabei signifikant, wie aus den Werten des Ljung/Box-Tests ${ }^{64}$ (Q-Statistisk, Portmanteau-Test) ersichtlich. Die entsprechenden $p$-Werte, d.h. Wahrscheinlichkeiten für eine Ablehnung der Nullhypothese (hier: signifikante Autokorrelationen für alle Ordnungen bis 36) sind sämtlich null. Auch die Tatsache, dass nur die par-

62 Zur Autokorrelationsfunktion vgl. etwa Buscher (2002, S. 146 f.).

63 Vgl. hierzu etwa die Illustration in Buscher (2002, S. 156) oder Hamilton (1994, S. 50).

64 Zum Ljung/Box-Test vgl. etwa Buscher (2002, S. 150 f.) oder Campbell et al. (1997, S. 47). 
tielle Autokorrelation ${ }^{65}$ erster Ordnung signifikant ist, deutet klar auf einen AR(1)-Prozess hin.

Kommen wir nun zum Korrelogramm der ersten Differenzen der KGV-Reihe, d.h. dem Korrelogramm des Prozesses $\left\{V_{t}-V_{t-1}\right\}$. Abbildung B.2 enthält die entsprechenden Werte.

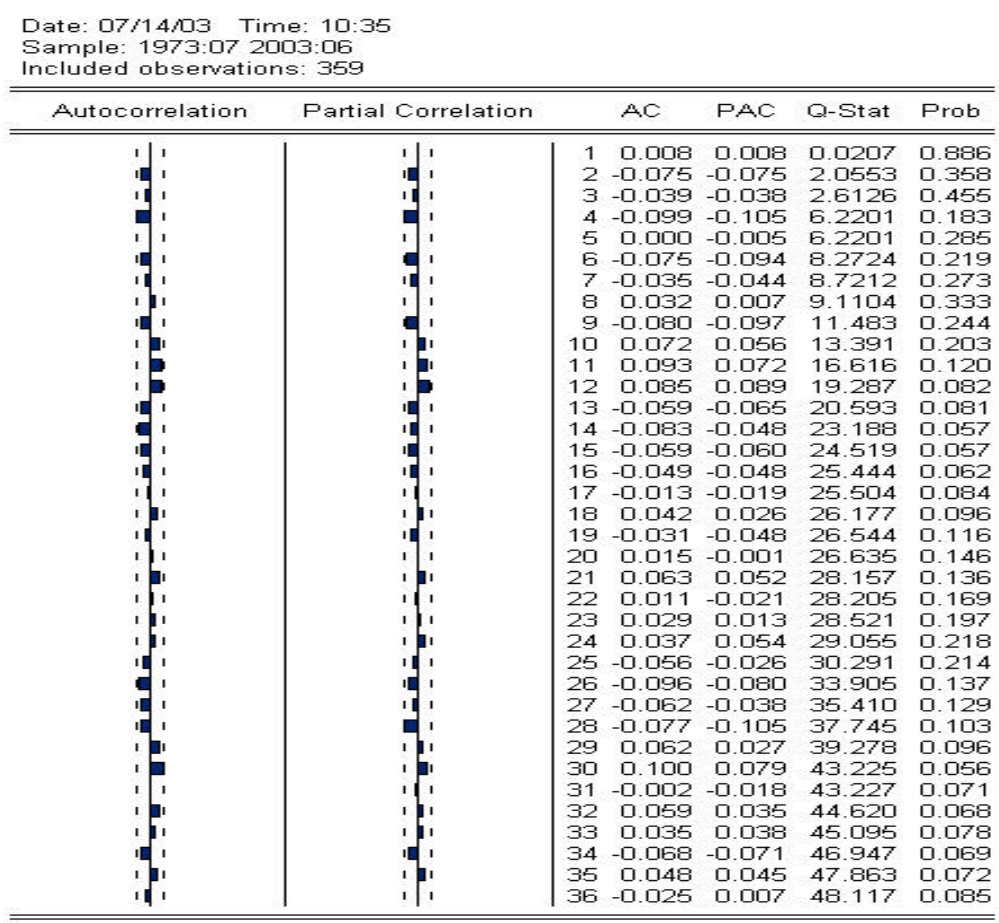

Abbildung B.2: Korrelogramm der ersten Differenzen der KGV-Zeitreihe 07/73 - 06/03

Es zeigt sich, dass die einzelnen Autokorrelationen zwar nicht signifikant (unter dem von EViews verwendeten Standard-Signifikanzniveau von 5\%) sind. Zieht man aber die bestehenden Korrelationen in ihrer Gesamtheit in Betracht, so liegt eine Signifikanz vor. Dies offenbart der Ljung/Box-Test (Q-Statistik). Die entsprechenden $p$-Werte zeigen, dass die Nullhypothese

$H_{0}: \quad$ Alle Autokorrelationen bis einschließlich der Ordnung $n$ sind null,

für alle Ordnungen bis $n=36$ sowohl zum 1\%- als auch zum 5\%-Signifikanzniveau abgelehnt wird. Nur zum 10\%-Signifikanzniveau gibt es einzelne Ordnungen, die nicht zur Verwerfung der Nullhypothese führen. Nimmt man das 5\%-Signifikanzniveau als standardmäßi-

65 Zur partiellen Autokorrelationsfunktion vgl. etwa Buscher (2002, S. 152 ff.) oder Hamilton (1994, S. 111 ff.). 
gen Maßstab, so spricht dieses Ergebnis eindeutig gegen das Vorliegen eines Random Walks, denn für diesen stellt die Zeitreihe der ersten Differenzen gemäß (A.7b) einen White NoiseProzess dar. Dies beinhaltet, dass sämtliche Autokorrelationen gleich null sein müssen.

\section{B.2 Spezifikation und Analyse der Basis-Regressionsgleichung}

Die Regressionsgleichung (A.10b) ist die Basisgleichung zur Spezifikation eines AR(1)Prozesses. Die Durchführung dieser Regression in EViews 4.1 führt zu den in Tabelle B.1 enthaltenen Ergebnissen.

\begin{tabular}{|c|c|c|c|c|}
\hline \multicolumn{5}{|c|}{$\begin{array}{l}\text { Dependent Variable: KGV_M } \\
\text { Method: Least Squares } \\
\text { Date: } 05 / 13 / 03 \text { Time: } 18: 13 \\
\text { Sample(adjusted): } 1973: 08 \text { 2003:06 } \\
\text { Included observations: } 359 \text { after adjusting endpoints }\end{array}$} \\
\hline Variable & Coefficient & Std. Error & t-Statistic & Prob. \\
\hline$\stackrel{C}{\mathrm{KGV}} \stackrel{\mathrm{M}(-1)}{ }$ & $\begin{array}{l}0.701539 \\
0.952538\end{array}$ & & & $\begin{array}{l}0.0050 \\
0.0000\end{array}$ \\
\hline $\begin{array}{l}\text { R-squared } \\
\text { Adjusted R-squared } \\
\text { S.E. of regression } \\
\text { Sum squared resid } \\
\text { Log likelihood } \\
\text { Durbin-Watson stat }\end{array}$ & $\begin{array}{r}0.906537 \\
0.906275 \\
1.153661 \\
475.1434 \\
-559.7117 \\
1.937526\end{array}$ & $\begin{array}{l}\text { Mean depel } \\
\text { S.D. depen } \\
\text { Akaike info } \\
\text { Schwarz cri } \\
\text { F-statistic } \\
\text { Prob(F-stati }\end{array}$ & $\begin{array}{l}\text { ent var } \\
\text { nt var } \\
\text { iterion } \\
\text { rion }\end{array}$ & $\begin{array}{l}14.85376 \\
3.768353 \\
3.129313 \\
3.150947 \\
3462.703 \\
0.000000\end{array}$ \\
\hline
\end{tabular}

Tab. B.1: Spezifikation der AR(1)-Regressionsgleichung für die KGV-Reihe 07/73 - 06/03

Zunächst ergibt sich, dass die identifizierte Regressionsgleichung die Form

$$
V_{t}=0.701539+0.952538 \cdot V_{t-1}+Z_{t}
$$

besitzt. Die Parameter $c=0.701539$ und $a=0.952538$ sind beide eindeutig signifikant. Der p-Wert gibt an, dass die Nullhypothese (hier: Parameterwerte ungleich null) selbst auf einem Konfidenzniveau von $0.5 \%$ verworfen werden muss. Der $R^{2}$-Wert für die Güte der Anpassung liegt sowohl unmodifiziert als auch modifiziert über 90\%, d.h. die Regression erklärt über 90\% der empirischen Gesetzmäßigkeit. Auch die Durbin/Watson-Statistik ${ }^{66}$ liegt sehr in der Nähe ihres Idealwertes von 2. Dies bedeutet, dass die Residuen nicht korreliert und die ausgewiesenen Schätzwerte somit valide sind. Auch die F-Statistik, die testet, ob die Regressi- 
onskoeffizienten simultan gleich null sind, führt zu einer Verwerfung dieser Annahme, der entsprechende $p$-Wert ist null.

Der Wert des Regressionskoeffizienten $a$ in Höhe von 0.952538 liegt unterhalb des kritischen Wertes von $a=1$, der im Falle eines Random Walk vorliegt, allerdings nicht sehr deutlich. Inwieweit der Wert $a=1$ statistisch signifikant unterschritten wird, wird im Rahmen der sog. Einheitswurzel bzw. Stationaritätstests untersucht; man vgl. hierzu die Ergebnisse in Albrecht/Kantar (2003).

Bringen wir nun die spezifizierte Regressionsgleichung in die äquivalente Form eines AR(1)Prozesses gemäß (A.10a). Aufgrund von $c=m(1-a)$ ergibt sich die folgende Repräsentation:

$$
V_{t}-14.8=0.952538\left(V_{t-1}-14.8\right)+Z_{t}
$$

Der langfristige Gleichgewichtswert des AR(1)-Prozesses ist damit ein KGV-Wert von $m=14.8$, auf den die empirischen $\mathrm{KGV}$-Werte im Zeitablauf hingezogen werden. Der langfristige Gleichgewichtswert liegt somit stark in der Nähe des Mittelwerts der KGV-Reihe in Höhe von 14.85. 


\section{Anhang C: Grundlagen einer DAX-Projektion}

Bezeichnet man mit $K_{t}$ den DAX-Kurs zum Zeitpunkt $t$ und mit $G_{t}$ den (akkumulierten) DAX-Gewinn zum Zeitpunkt $t$, so ergibt sich das Kurs/Gewinn-Verhältnis $K G V_{t}$ zum Zeitpunkt $t$ zu $K G V_{t}=K_{t} / G_{t}$. Für die relative Kursveränderung zweier aufeinander folgender Perioden gilt dann

$$
\frac{K_{t+1}}{K_{t}}=\frac{K G V_{t+1}}{K G V_{t}} \cdot \frac{G_{t+1}}{G_{t}}
$$

und damit

$$
K_{t+1}=\frac{K G V_{t+1}}{K G V_{t}} \cdot \frac{G_{t+1}}{G_{t}} \cdot K_{t}
$$

Der projizierte DAX-Stand hängt somit ab vom KGV-Wachstum, vom Gewinnwachstum und vom angenommenen Startwert des DAX. Ausgangskurs $K_{t}$ und Ausgangs-KGV $K G V_{t}$ sind bekannt. Als KGV zum Zeitpunkt $t+1$ wäre das Gleichgewichts-KGV in Höhe von $14.8 \mathrm{zu}$ wählen. Offen ist damit nur noch die Substantiierung der Gewinnwachstumsrate. Diese kann man auf Basis der Beziehung $G_{t}=K_{t} / K G V_{t}$ aus den entsprechenden Werten der DatastreamDatenbank für die Entwicklung des DAX sowie des DAX-KGVs berechnen, man vgl. das entsprechende Ergebnis in Abschnitt 4 des Haupttextes. In vollkommener Analogie zu (C.2) kann auch eine mehrperiodige DAX-Projektion durchgeführt werden. Im Falle eines Gewinnwachstums von null, d.h. $G_{t+1}=G_{t}$ reduziert sich (C.2) auf

$$
K_{t+1}=K G V_{t+1} \cdot \frac{K_{t}}{K G V_{t}}
$$

Dies entspricht dem in Abschnitt 4 des Haupttextes dargestellten Fall einer "Umrechnung" des aktuellen DAX-Wertes unter Ansatz eines Gleichgewichts-KGV. 


\section{Literatur}

Albrecht, P. (2001): Welche Aktienperformance ist über die nächsten Dekaden realistischerweise zu erwarten? Eine Fundamentalanalyse, Zeitschrift für Versicherungswesen 23/2001, $803-812$.

Albrecht, P. (2003): Produktgarantien und Aktienkrise - Implikationen für die Kapitalanlagepolitik von Lebensversicherungsunternehmen, erscheint in: Zeitschrift für die gesamte Versicherungswissenschaft 2003. [Download unter: www.bwl.uni-mannheim.de/Albrecht; dort unter: Forschung/Schriftenreihen/Mannheimer Manuskripte/Manuskript Nr. 148].

Albrecht, P., C. Kantar (2003): Random Walk oder Mean Reversion? Eine statistische Analyse auf fundamentaler Basis für den deutschen Aktienmarkt, Universität Mannheim.

Albrecht, P., R. Maurer (2002): Investment- und Risikomanagement, Stuttgart.

Balvers, R., Y. Wu, E. Gilliland (2000): Mean Reversion Across National Stock Markets and Contrarian Investment Strategies, Journal of Finance 55, 745 - 772.

Buscher, H.S. (2002): Angewandte Zeitreihenanalyse, in: Schröder, M. (Hrsg.): FinanzmarktÖkonometrie, Stuttgart, S. $131-212$.

Campbell, J.Y., A.W. Lo, A.C. MacKinlay (1997): The Econometrics of Financial Markets, Princeton, New Jersey.

Campbell, J.Y., L.M. Viceira (1999): Consumption and Portfolio Decisions when Expected Returns are Time Varying, Quarterly Journal of Economics 114, 433 - 495.

Campbell, J.Y., L.M. Viceira (2002): Strategic Asset Allocation, Oxford, New York.

Coenenberg, A.G. (2003): Jahresabschluss und Jahresabschlussanalyse, 19. Aufl., Stuttgart.

Eberts, E. (2003): The connection of stock markets between Germany and the USA, ZEW Discussion Paper No. 03-36, Zentrum für Europäische Wirtschaftsforschung, Mannheim.

Fama, E.F. (1976): Efficient Capital Markets: Reply, Journal of Finance 31, 143 - 145.

Fama, E.F., K. French (1988): Permanent and Temporary Components of Stock Prices, Journal of Political Economy 96, 246 - 273.

Financial Accounting Standards Board (Hrsg., 1995): Statement of Financial Accounting Standards No. 87, Employers' Accounting for Pensions, Norwalk, Connecticut.

Franke, J., W. Härdle, C. Hafner (2001): Einführung in die Statistik der Finanzmärkte, Berlin, Heidelberg.

Granger, C.W.J. (1969): Prediction with a generalized cost of error function, Operations Research Quarterly 20, 199 - 207.

Granger, C.W.J., P. Newbold (1977): Forecasting Economic Time Series, New York u.a. 
Gujarati, D.N. (1995): Basic Econometrics, 3. Aufl., New York u.a.

Hamilton, J.D. (1994): Time Series Analysis, New Jersey.

Küting, K., C.-P. Weber (2001): Die Bilanzanalyse, 6. Aufl., Stuttgart.

Küting, K., C.-P. Weber (2003): Handbuch der Rechnungslegung, Einzelabschluss, 5. Aufl., Stuttgart.

LeRoy, S. (1973): Risk Aversion and the Martingale Property of Stock Returns, International Economic Review 14, 436 - 446.

LeRoy, S. (1989): Efficient Capital Markets and Martingales, Journal of Economic Literature $27,1583-1621$.

Lo, A. (Hrsg., 1996): Market Efficiency: Stock Market Behaviour in Theory and Practice, London.

Lo, A.W., A.C. MacKinlay (1988): Stock Market Prices Do Not Follow Random Walks: Evidence from a Simple Specification Test, Review of Financial Studies 1, 41 - 46.

Lucas, R., Jr. (1978): Asset Prices in an Exchange Economy, Econometrica 46, 1429 - 1446.

McCarthy, D., (2003): Buchbesprechung zu Strategic Asset Allocation by John Y. Campbell \& Luis M. Viceira, in: Journal of Pension Economics and Finance 2, 93 - 94.

Perridon, L., M. Steiner (2002): Finanzwirtschaft der Unternehmung, 11. Aufl. München.

Poddig, T., H. Dichtl, K. Petersmeier (2000): Statistik, Ökonometrie, Optimierung, Bad Soden/Ts.

Poterba, J.M., L.H. Summers (1988): Mean Reversion in Stock Returns: Evidence and Implications, Journal of Financial Economics 22, 27 - 59.

Samuelson, P.A. (1965): Proof that Properly Anticipated Prices Fluctuate Randomly, Industrial Management Review 6, 41 - 49.

Samuelson, P.A. (1973): Proof that Properly Discounted Present Values of Assets Vibrate Randomly, Bell Journal of Economics and Management Science 4, 369 - 374.

Shiller, R., P. Perron (1985): Testing the random walk hypothesis, Economic Letters 18, 381 -386 .

Steiner, M., C. Bruns (2002): Wertpapiermanagement, 8. Aufl., Stuttgart. 